\title{
Cell and gene therapy for carbamoyl phosphate synthetase I deficiency
}

\begin{abstract}
Carbamoyl phosphate synthetase 1 (CPS1) is the first and rate-limiting enzyme in the urea cycle. CPS1 deficiency is a devastating condition, which is clinically characterized by periodic episodes of life-threatening hyperammonemia. Currently, there is no cure for CPS1 deficiency except for liver transplantation, which is limited by a severe shortage of donors and significant risk of mortality and morbidity. Based on the progress to date, cell-based therapies-including hepatocyte or stem cell transplantation-and new approaches for gene therapy have become the promising curative treatments for CPS1 deficiency. This review outlines the current progress and challenges of cell and gene therapies for CPS1 deficiency.
\end{abstract}

Keywords: urea cycle defects, carbamoyl phosphate synthetase 1 deficiency, cell therapy, gene therapy
Volume 7 Issue I - 2017

\author{
Yuang Zhang,' Bin $\mathrm{Li}^{2}$ \\ 'Associate at Department of Genetic Medicine, Children's \\ Research Institute, Children's National Health System, USA \\ ${ }^{2}$ Washington Institute for Health Sciences, Department of \\ Biochemistry and Molecular \& Cellular Biology, Georgetown \\ University Medical Center, USA
}

Correspondence: Bin Li, MD, Washington Institute for Health Sciences, 460I N Fairfax Drive, Arlington,VA 22203; Georgetown University Medical Center, 4000 Reservoir Road, N.W.,Washington D.C. 20057, United States, Tel 202-687-6484, Fax (202) 687-1800, Email bl444@georgetown.edu

Received: July 03, 2017 | Published: July 14, 2017
Abbreviations: AAVs, adeno-associated viruses; ADA, adenosine deaminase; ADHLSCs, adult-derived human liver stem cells; ASL, argininosuccinate lyase; ASS, argininosuccinate synthetase; CPS1, carbamoyl phosphate synthetase 1; CRISPR/Cas9, clustered, regularly interspaced, short palindromic repeats/crisprassociated protein 9; ESCs, embryonic stem cells; iPSC, induced pluripotent stem cell; iHLCs, iPSC-derived hepatocyte-like cells; NAGS, n-acetylglutamate synthase; NCG, n-carbamyl-1-glutamate; OAs, organic acidemias; OLT, orthotopic liver transplantation; OTC, ornithine transcarbamylase; UCDs, urea cycle defects

\section{Introduction}

The urea cycle is a series of biochemical reactions that occurs in the liver and converts highly toxic ammonia to urea. There are six enzymes involved in this cycle, including $\mathrm{N}$-acetylglutamate synthase (NAGS), carbamyl phosphate synthetase 1 (CPS1), ornithine transcarbamylase (OTC), arginase, argininosuccinate synthetase (ASS), and argininosuccinate lyase (ASL). Urea cycle defects (UCDs) are a group of inherited diseases caused by a mutation that results in a deficiency in one of the above six enzymes. In these conditions, the excess ammonia produced from breaking down protein accumulates in the body and causes toxic effects, especially for the central nervous system. CPS1 is the first and rate-limiting enzyme in the urea cycle. The cytogenetic location of CPS1 gene is at $2 \mathrm{q} 34$, which is the long (q) arm of chromosome 2 at position 34 (Homo sapiens Annotation Release 108, GRCh38.p7) (NCBI). CPS1 deficiency is one of the less frequent UCDs and is caused by a complete or partial deficiency in the enzyme CPS1. This disease is an autosomal recessive metabolic disorder, which means both copies of the gene in each cell have mutations. If an individual only carry one copy of the mutated gene, he or she typically do not show signs and symptoms of CPS1 deficiency. This condition often is found in newborns but may be diagnosed in a few less severely affected patients later in life. Until now, more than 240 CPS1-associated genetic changes have been identified. ${ }^{1}$ CPS1 deficiency is a rare disease with an incidence of approximately 1 to 9 in 1,000,000 (http://www.orpha.net/consor/www/cgi-bin/OC_Exp. php?lng=EN\&Expert=147) and 1/1,300,000 live births in the USA. ${ }^{2}$
Infection with fever or any other physical stressor, such as injury or surgery, can cause lethal hyperammonemic crises in neonates or young infants with CPS1 deficiency. The symptoms of neonatal-onset form of CPS1 deficiency include lethargy, unwillingness to feed, vomiting, hypothermia, hypotonia, seizures, coma, and can lead to death. Beyond the newborn period, patients can present hyperammonemia with irritability, lethargy, headache, seizures, confusion and cognitive disability.

Currently, there is no cure for CPS1 deficiency except for liver transplantation. To avoid acute hyperammonemia, which is the major treatment focus for this disease, additional key therapeutic strategies include promoting toxic ammonia excretion and reducing dietary protein intake/optimizing caloric intake. Hemodialysis and ammonia scavenger medications (sodium benzoate, sodium phenylbutyrate, arginine, citrulline) can facilitate ammonia excretion. A balanced diet can limit excess dietary protein, reverse endogenous catabolism and decrease the excess ammonia produced from the breakdown of protein. ${ }^{1}$ Other experimental therapeutic approaches are also under study. Recently, basic studies have shown that N-carbamyl-Lglutamate (NCG), the chemical analog of CPS1's essential activator, can improve and protect the function of mutant CPS1. Clinical studies have also shown that NCG had beneficial effects on CPS1-deficient patients with some special mutations, such as the p.Glu1034Gly mutation. ${ }^{3}$ The treatment outcomes depends on disease severity. About $50 \%$ of children with newborn onset UCDs did not survive to age 5 years. Neonatal-onset CPS1 deficiencies seemed to have a worse prognosis. Episodes of hyperammonemic coma of long duration are associated with cognitive disability (http://www.nucdf.org/ucd faqs. htm).

In patients whose symptoms cannot be controlled by the treatments described above, orthotopic liver transplantation (OLT) is the final curative option. Data from the United Network for Organ Sharing for 2002-2012 described 293 patients with UCDs and organic acidemias (OAs) who received liver transplantation. Within 5 years after transplantation, the graft survival rate was $78 \%$ for children $<2$ years old at the time of transplantation and $88 \%$ for children $\geq 2$ years old at the time of transplantation. ${ }^{4}$ Foschi et al. summarized that the 
advantages of orthotopic liver transplants in children with UCDs are an approximate $90 \%$ survival rate, normal mental development, and a better quality of life. ${ }^{5}$ However, whole liver transplantation for these disorders is limited by a severe shortage of donors, the risks associated with major surgery, and the requirement for lifelong immunosuppressive therapy. Currently, approximately 17,000 adults and children are waiting for donated livers in United States, and the waiting list continues to grow. Every year, more than 1,500 people die waiting for a donated liver to become available (http://www. liverfoundation.org/patients/organdonor/about/). Therefore, there is certainly an urgent need for novel curative therapies. Based on the progress to date, cell-based therapies and new approaches in gene therapy have become the promising curative treatments for CPS1 deficiency. This review outlines the current progress and challenges of cell and gene therapies for CPS1 deficiency.

\section{Cell therapies for CPS I deficiency}

Hepatocyte transplantation was proposed as a treatment for metabolic diseases of the liver in the 1990s. ${ }^{6}$ Compared with orthotopic liver transplantation, hepatocyte transplantation has some advantages, including the relatively safe surgical procedure, the ability to share the donor's hepatocytes among several patients, and the long-term cryopreservation of hepatocytes. ${ }^{7}$ Michel et al. studied hepatocyte transplantation as an alternative therapy in a UCD mouse model and found that functional transplanted hepatocytes could survive in the spleen for over 5 months after transplantation in syngeneic hepatocyte transplantations (C57 mice to C57 mice). However, in allogeneic transplantations (C57 mice to $\mathrm{spf}{ }^{\text {ash }}$ mice) without immunosuppression, hepatocytes were rejected in 3 days. With immunosuppression, hepatocytes survived 12 days and showed OTC activity, but the metabolic disorder was not corrected. This study demonstrated that rejection in allogeneic transplantation is the major problem. $^{8}$

As Meyburg et al. reported, a 10-week-old boy with CPS1deficiency received a transplantation of cryopreserved hepatocytes. During the 11 months after hepatocyte transplantation, he never developed life-threatening metabolic crises. Plasma urea increased from $18 \pm 9 \mathrm{mg} / \mathrm{dL}$ to $25 \pm 8 \mathrm{mg} / \mathrm{dL}$. Protein intake was increased 5 months after hepatocyte transplantation but was lowered 2 months later because of hyperalimentation and extensive weight gain. The authors concluded that although hepatocyte transplantation is not a permanent therapeutic option, it is suitable to bridge patients until liver transplantation..$^{9}$ A group of 10 patients with UCDs who had undergone hepatocyte transplantation was summarized by 2011. Most of these cases had very few technical complications and encouraging clinical results. ${ }^{10}$

According to the database Clinicaltrials.gov, there are two clinical trials studying hepatocyte transplantations. One is an open, prospective, uncontrolled, multicenter study to evaluate the safety and efficacy of a liver cell suspension infused into the portal vein of children with UCDs. ${ }^{11}$ In this study, neonates and infants within the first 3 months of life with UCDs were treated by repetitive application of human liver cells. The aim of this new therapeutic option is to supply a sufficient amount of healthy liver cells to compensate for the metabolic defect and to reduce the risk of neurological deterioration while awaiting OLT. Another is a single-center study at Children's Hospital of Pittsburgh at the University of Pittsburgh Medical Center. ${ }^{12}$ The purpose of this clinical study is to determine whether partial irradiation of the liver and subsequent liver cell transplantation can provide help for patients with life-threatening liver-based metabolic diseases, including UCDs.
In this study, the improvement in enzyme physiologic function will be assessed 6 months after infusing allogeneic donor hepatocytes through the portal vein following preparative hepatic irradiation. Currently, there are no study results posted.

The major challenges for hepatocyte transplantation are the low rates of engraftment (from $0 \%$ to a maximum of $12 \%$ ) and short-term survival of transplanted hepatocytes due to rejection or senescence. Most patients lose their graft after several months to years. In addition, the lack of good-quality donor organs is another major limitation. Therefore, increasing engraftment rates and liver repopulation are important research topics for hepatocyte therapy. ${ }^{10}$

The use of stem cells is a hot research topic, since the availability of hepatocytes is limited, while stem cells could be expanded in vitro and differentiated to functional liver cells. There are several kinds of liver stem cells that are under investigation. Embryonic stem cells (ESCs) are omnipotent stem cells. Pluripotent stem cells include fetal hepatoblasts, fetal hepatic progenitor cells, hematopoietic stem cells, adipose-derived stem cells, amniotic epithelial cells, umbilical cord blood cells and mesenchymal stem cells. Oval cells in the adult liver are a type of bipotent cell that can differentiate into hepatocytes and bile duct epithelial cells. Adult hepatocytes can be called unipotent liver stem cells, since they can proliferate during liver regeneration. ${ }^{10}$

As Lombard et al. reported, adult-derived human liver stem cells (ADHLSCs) seem to have clinical effects for the treatment of UCDs. They found that ADHLSC-derived extracellular vesicles contained mRNA encoding some of the enzymes deficient in UCDs, including CPS1 and are capable of transferring their mRNA contents to recipient cells. mRNA transfer via extracellular vesicles may therefore be one of the modes of action of ADHLSCs in the treatment of UCDs (https:// dial.uclouvain.be/pr/boreal/object/boreal:184885).

\section{Gene therapies for CPSI deficiency}

Gene therapy involves delivering genetic material into patients' cells to fight or prevent disease. This involves replacing an abnormal gene with a normal gene, inactivating or knocking out a mutated gene, or introducing a new gene to fight a disease. This technology is still young, but it has been used with some success. Currently, there are 5240 studies related to gene therapy listed on the database Clinicaltrials. gov. On September 14, 1990, the first FDA approved gene therapy procedure was carried out on a 4-year-old girl, Ashanti DeSilva, with Adenosine Deaminase (ADA) deficiency. This procedure was not a cure, as the therapy needs to be repeated every two years. She attended college and is still in good health with a normal life. In 2003, the first commercial gene therapy medicine (Gendicine) carrying the p53 tumor-suppressor gene was available on the market in China. In November 2012, the European Commission approved the gene therapy Glybera, which is used to treat Familial Hyperchylomicronemia. This is the first gene therapy drug approved by regulatory authorities in the Western world. ${ }^{13}$ Personalized medicine (or precision medicine) is a medical model in which the genetic information plays a major role for the customization of healthcare. Personalized gene therapy is the most cutting-edge treatment method, especially for monogenic disorders. Most monogenic disorders only have very rare cases; however, they have variable mutations for single genes. In fact, more than $90 \%$ of the known CPS1 mutations are private. ${ }^{14}$

Mouse models exist for each of the UCDs except for NAGS deficiency, and the clinical and biochemical phenotypes of these mouse models closely resemble the phenotypes seen in human patients. Therefore, all the current mouse models are highly useful 
for gene therapy studies. ${ }^{15}$ To study gene therapies directed to the hepatocytes to treat CPS1 deficiency, Schofield et al. generated a CPS1 deficient mouse by gene targeting. They found that the mice with homozygous disruption of CPS1 (CPSase. ${ }^{-/}$mice) die within 36 hours of birth with overwhelming hyperammonemia. The plasma ammonia was undetectable in CPSase $(+/+)$ mice killed within 6 hours of birth. Blood ammonia concentration was 142 to $365 \mu \mathrm{mol} / \mathrm{L}$ in CPSase (+/-) mice and 3,616 to 5,630 $\mu \mathrm{mol} / \mathrm{L}$ in CPSase (-/-) mice within 6 hours of birth. The authors believe it is a good model of human CPS1 deficiency. ${ }^{16}$

Due to the disease prevalence and severity in humans, the majority of pre-clinical gene therapy studies have focused on OTC deficiency. ${ }^{17}$. In addition, the partially OTC-deficient sparse fur mouse ( $\mathrm{spf}^{\text {ash }}$ mouse) is an authentic model for the gene therapy study of OTC deficiency, since the colony of spf $f^{\text {ash }}$ mice can be easily maintained, and they can express $5 \%$ to $15 \%$ wild-type levels of active enzyme. ${ }^{18}$

Ye et al. used a recombinant adenovirus containing the normal murine homolog of OTC cDNA with a strong constitutive promoter from cytomegalovirus to treat $\mathrm{spf}^{\text {ash }}$ mice. They found that liver OTC enzyme activity in this mouse model was completely recovered and persisted for at least 2 months. The serum glutamine and urine orotic acid were also transiently corrected..$^{19}$ Raper et al. also studied gene therapy for OTC deficiency with an adenoviral vector in the spf fash mouse. To blunt the cytotoxic $\mathrm{T}$ lymphocyte immune response to the vector and vector-transduced cells, they modified the adenoviral construct and created an E1 deletion-E2 temperaturesensitive mutation. They treated the $\mathrm{spf}^{\text {ash }}$ mouse with the modified recombinant adenovirus containing the OTC cDNA and found that biochemical abnormalities of model mice were corrected for 2-3 months and that the transduction and expression of OTC occurred within 24 hours after infusion. Subsequent preclinical studies in mice and non-human primates demonstrated that the modified recombinant adenovirus had a substantially improved safety profile and similar efficacy. ${ }^{20}$ However, the subsequent phase 1 clinical trial in adults with partial OTC deficiency failed due to the lethal inflammatory response in a subject in 1999. In this study, they found that the transduced OTC gene was expressed in the hepatocytes of 7 out of 17 subjects. Three out of 11 subjects with symptoms related to OTC deficiency showed modest increases in urea cycle metabolic activity, but these were not statistically significant. ${ }^{21}$

This fatal accident put the entire field of gene therapy on hold for many years. Recently, the adeno-associated viruses (AAVs) have been emerging as a highly effective and safe gene delivery system. Glybera, the first gene therapy product approved in the European Union, is an adeno-associated viral vector engineered to express lipoprotein lipase. ${ }^{13}$ Among the AAV serotypes, AAVs 2 and 8 have been shown to be the most efficient for liver-directed gene therapy. Pioneering liverdirected gene therapy trials for hemophilia B have achieved sustained clinical improvement after a single systemic injection of adenoassociated virus (AAV 2/8) derived vectors encoding the human factor IX cDNA.22

Wang et al. generated a clinical candidate vector for a proposed OTC gene therapy trial in humans based on a self-complementary AAV8 vector expressing codon-optimized human OTC (hOTCco) under the control of a liver-specific promoter. They found that the expression levels and activity of hOTC were higher than physiological levels in adult spf ${ }^{\text {ash }}$ mice following a single intravenous injection of hOTCco vector. Vector doses at $1 \times 1010$ genome copies achieved sustained correction of the OTC deficiency biomarker orotic aciduria and clinical protection against an ammonia challenge. ${ }^{23}$ Alexander et al. also successfully cured OTC-deficient mice using an AAV8-based gene therapy approach, ${ }^{24}$ but later found that AAV8 is far less effective for gene delivery to human liver cells. A novel AAV, referred to as LK03, which targets human liver cells with a much higher efficiency than AAV8 (12 times) has been developed. A preclinical study of the $\mathrm{AAV} / \mathrm{LK}$ 03-based gene delivery system for the treatment of OTC deficiency is ongoing at University College London, which will likely lead to the authorization of a phase I/II AAV/LK03-mediated clinical trial for OTC deficiency in pediatric patients (http://gtr.rcuk.ac.uk/ projects? ref $=\mathrm{MR} \% 2 \mathrm{FN} 019075 \% 2 \mathrm{~F} 1$ )

The novel Clustered, Regularly Interspaced, Short Palindromic Repeats/CRISPR-associated protein 9 (CRISPR/Cas9) system is an attractive genome-editing method that can be used to correct a known mutation. Sin et al. used CRISPR/Cas9 with piggyBac technology to restore the function of arginase- 1 in induced pluripotent stem cell (iPSC)-derived hepatocyte-like cells (iHLCs) and macrophages from a tamoxifen-inducible arginase- 1 deficient mouse model. The target gene was successfully repaired in the iHLCs, and these cells showed minimal urea cycle function. The iPSC-derived macrophages also expressed substantial amounts of arginase. They believe that their studies provide proof-of-concept for gene editing in UCDs. ${ }^{25}$

Regarding gene therapy for CPS1 deficiency, Lipshutz et al. have developed a novel conditional CPS1-deficient knockout mouse. They propose to develop methods for gene correction of CPS1 deficiency with recombinant helper-dependent adenoviral vectors and adeno-associated viral vectors by neonatal administration in this CPS1-deficient mouse model http://grantome.com/grant/NIH/R21NS091654-01).

\section{Conclusion}

Cell and gene therapies are now emerging as promising curative treatments for rare genetic metabolic disorders, such as CPS1 deficiency. Although there are many challenges, especially regarding the safety of clinical trial participants, with the development of precision medicine it can be reasonably predicted that gene therapy or an optimal combination of cell and gene therapy could be the final way to end rare genetic disorders of metabolism, including CPS1 deficiency.

\section{Acknowledgements}

The authors are grateful to Dr. Gengxiang Zhao and Dr. Dashuang Shi's expert advices for this project. The authors were supported for this work by the Washington Institute for Health Sciences.

\section{Conflicts of interest}

The authors declare no conflict of interest.

\section{References}

1. Diez-Fernandez C, Häberle J. Targeting CPS1 in the treatment of Carbamoyl phosphate synthetase 1 (CPS1) deficiency, a urea cycle disorder. Expert Opin Ther Targets. 2017;21(4):391-399.

2. Summar ML, Koelker S, Freedenberg D, et al. The incidence of urea cycle disorders. Mol Genet Metab. 2013;110(1-2):179-180.

3. Shi D, Zhao G, Ah Mew N, et al. Precision medicine in rare disease: Mechanisms of disparate effects of N-carbamyl-1-glutamate on mutant CPS1 enzymes. Mol Genet Metab. 2016;120(3):198-206.

4. Perito ER, Rhee S, Roberts JP, et al. Pediatric liver transplantation for urea cycle disorders and organic acidemias:United Network for Organ Sharing data for 2002-2012. Liver Transpl. 2014;20(1):89-99. 
5. Foschi FG, Morelli MC, Savini S, et al. Urea cycle disorders: A case report of a successful treatment with liver transplant and a literature review. World J Gastroenterol. 2015;21(13):4063-4068.

6. Strom SC, Fisher RA, Rubinstein WS. Transplantation of human hepatocytes. Transplant Proc. 1997;29(4):2103-2106.

7. Jorns C, Ellis EC, Nowak G, et al. Hepatocyte transplantation for inherited metabolic diseases of the liver. J Intern Med. 2012;272(3):201223.

8. Michel JL, Rabier D, Rambaud C, et al. Intrasplenic transplantation of hepatocytes in spf-ash mice with congenital ornithine transcarbamylase deficiency. Chirurgie. 1993;119(10):666-671.

9. Meyburg J, Das AM, Hoerster F, et al. One liver for four children:first clinical series of liver cell transplantation for severe neonatal urea cycle defects. Transplantation. 2009;87(5):636-641.

10. Meyburg J, Hoffmann GF. Liver, liver cell and stem cell transplantation for the treatment of urea cycle defects. Mol Genet Metab. 2010;100 Suppl 1:S77-S83.

11. https://clinicaltrials.gov/ct2/show/NCT00718627

12. https://clinicaltrials.gov/ct2/show/NCT01345578.

13. Wirth T, Parker N, Ylä-Herttuala S. History of gene therapy. Gene. 2013;525(2):162-169.

14. Häberle J, Shchelochkov OA, Wang J, et al. Molecular defects in human carbamoy phosphate synthetase I:mutational spectrum, diagnostic and protein structure considerations. Hum Mutat. 2011;32(6):579-589.

15. Deignan JL, Cederbaum SD, Grody WW. Contrasting features of urea cycle disorders in human patients and knockout mouse models. $\mathrm{Mol}$ Genet Metab. 2008;93(1):7-14.

16. Schofield JP, Cox TM, Caskey CT, et al. Mice deficient in the urea-cycle enzyme, carbamoyl phosphate synthetase I, die during the early neonatal period from hyperammonemia. Hepatology. 1999;29(1):181-185.
17. Alexander IE, Kok C, Dane AP, et al. Gene therapy for metabolic disorders: an overview with a focus on urea cycle disorders. $J$ Inherit Metab Dis. 2012;35(4):641-645.

18. Hodges PE, Rosenberg LE. The spf ${ }^{\text {ash }}$ mouse:a missense mutation in the ornithine transcarbamylase gene also causes aberrant mRNA splicing. Proc Natl Acad Sci USA. 1989;86(11):4142-4146.

19. Ye X, Robinson MB, Batshaw ML, et al. Prolonged metabolic correction in adult ornithine transcarbamylase-deficient mice with adenoviral vectors. J Biol Chem. 1996;271(7):3639-3646.

20. Raper SE, Wilson JM, Yudkoff M, et al. Developing adenoviral-mediated in vivo gene therapy for ornithine transcarbamylase deficiency. $J$ Inherit Metab Dis. 1996;21(Suppl 1):119-137.

21. Raper SE, Yudkoff M, Chirmule N, et al. A pilot study of in vivo liverdirected gene transfer with an adenoviral vector in partial ornithine transcarbamylase deficiency. Hum Gene Ther. 2002;13(1):163-175.

22. Baruteau J, Waddington SN, Alexander IE, et al. Gene therapy for monogenic liver diseases:clinical successes, current challenges and future prospects. J Inherit Metab Dis. 2017;40(4):497-517.

23. Wang L, Morizono H, Lin J, et al. Preclinical evaluation of a clinical candidate AAV8 vector for ornithine transcarbamylase (OTC) deficiency reveals functional enzyme from each persisting vector genome. Mol Genet Metab. 2012;105(2):203-211.

24. Alexander IE, Kok C, Dane AP, et al. Gene therapy for metabolic disorders: an overview with a focus on urea cycle disorders. $J$ Inherit Metab Dis. 2012;35(4):641-645.

25. Sin YY, Price PR, Ballantyne LL, et al. Proof-of-Concept Gene Editing for the Murine Model of Inducible Arginase-1 Deficiency. Sci Rep. 2017;7(1):2585. 